\section{Creencias culturales sobre los médicos y percepción de discriminación: El impacto en la continuidad de la atención}

\author{
MARÍA JOSÉ BAEZA-RIVERA ${ }^{1, \mathrm{a}, \mathrm{b}}$, HÉCTOR BETANCOURT ${ }^{2,3, \mathrm{a}, \mathrm{b}}$, \\ NATALIA SALINAS-OÑTE ${ }^{2, \mathrm{a}, \mathrm{b}}$, MANUEL S. ORTIZ ${ }^{2,4, \mathrm{a}, \mathrm{b}}$
}

\section{Health care effects of cultural beliefs about physicians and perceived discrimination}

Background: Healthcare inequities may hamper physical and mental health. Aim: To examine perceived discrimination in healthcare services in relation to socio-structural and cultural antecedents as well as their effect on psychological processes and health. Material and Methods: Questionnaires on beliefs about physicians, perceived discrimination, emotions and affective states and avoidance consequences in health were answered by 337 child caregivers (85\% women) attending preventive health care appointments at primary health care centers. Results: Negative beliefs about healthcare professionals are directly associated with avoidance behaviors in health and perceived discrimination. The latter perception has no direct effects on avoidance behaviors, but it has an indirect effect through negative emotions associated with discrimination. Conclusions: There is an association between cultural, psychological and structural factors in health care. These results contribute to understand the phenomenon of discrimination and its negative consequences.

(Rev Med Chile 2019; 147: 161-167)

Key words: Culture; Discrimination; Emotions; Health Behavior; Physicians.

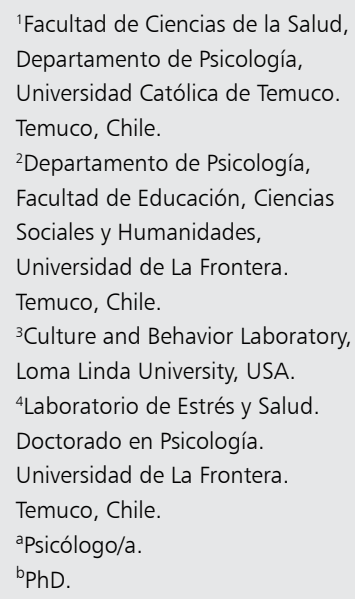

Recibido el 16 de octubre de 2018, aceptado el 4 de marzo de 2019.

\section{Correspondencia a:}

María José Baeza Rivera Carrera de Psicología Universidad Católica de Temuco, Manuel Montt 56, Temuco, Chile. maria.baeza@uct.cl
U no de los problemas más importantes en Latinoamérica es el de la desigualdad en salud ${ }^{1}$, lo que en Chile es visible en la discriminación o trato injusto en salud ${ }^{2}$. En línea con esto, la encuesta PNUD-DES da cuenta que el $41 \%$ de la población chilena declaró haber recibido alguna forma de mal trato, como ser "pasado a llevar" (30\%), ser "mirado en menos" (29\%), ser "discriminado" o "tratado injustamente" (27\%), situación que ha aumentado en los últimos quince años de $52 \%$ a $68 \%{ }^{3}$.

Las desigualdades en salud generan una asociación más fuerte entre la discriminación percibida e indicadores de salud física ${ }^{4,5}$, problemas de hipertensión arterial ${ }^{6} \mathrm{y}$ desenlaces en salud negativos, tales como mayor mortalidad ${ }^{7}$. En relación a la salud mental, las personas que indican haber sentido discriminación presentan mayor depresión, manía/hipomanía, desórdenes de estrés post traumático, trastorno de ansiedad generalizada y fobia social ${ }^{8-10}$.

Otra forma en que las desigualdades impactan el cuidado de la salud, es a través del acceso a las prestaciones médicas ${ }^{11}$. Se ha documentado que la percepción de discriminación y el maltrato en servicio de salud se asocia con una menor adherencia terapéutica ${ }^{2}$, asistencia a las citas médicas $^{12}$, disposición a utilizar los servicios preventivos de salud ${ }^{13-16}$, demora en la búsqueda de atención y medicamentos ${ }^{12,14,16-20}$, y la sustitu- 
ción de la atención convencional por la medicina alternativa ${ }^{21}$.

La discriminación viene asociada a emociones y estados afectivos, las que también se relacionan con conductas de salud. La evidencia sugiere que las emociones afectan los encuentros clínicos y las conductas de cuidado de la salud, ya que son un componente importante de las interacciones entre médicos y pacientes ${ }^{22}$ y se pueden ver afectadas por las diferencias culturales y las prácticas asociadas a las relaciones interpersonales ${ }^{23}$. Por ejemplo, el sentirse menoscabado, la falta de comprensión por parte de los médicos y los largos tiempos de espera se relacionan con indiferencia e irritabilidad $^{3}$. Estudios en Estados Unidos de Norteamérica con mujeres demuestran que la percepción de maltrato influye negativamente en la frecuencia y continuidad de los chequeos preventivos para el cáncer de mama $^{24}$, pero cuando esto va acompañado de emociones negativas tienen un impacto aún mayor en las mujeres latinas ${ }^{22-27}$.

Otro factor importante que permite explicar las conductas de cuidado de la salud son las creencias socialmente compartidas (culturales) asociadas a los profesionales ${ }^{28}$. Tales creencias, generalmente originadas en las experiencias previas con el sistema y profesionales de la salud, influyen la interacción con los profesionales y el cuidado de la salud. En este sentido las creencias, valores, normas y expectativas que tienen las personas sobre los profesionales de la salud pueden afectar la relación que se establece entre médico-paciente ${ }^{26}$. Desde este punto de vista, estudios han documentado diversas creencias negativas sobre los profesionales. Por ejemplo, estudios en Norteamérica con mujeres latinas identificaron creencias culturales que señalan a los profesionales como "severos y fríos"29, "poco confiables"30, "faltos de preocupación y compasión" ${ }^{31}$. Otros estudios en Chile con pacientes usuarias del sistema público señalan que los médicos son "impersonales", "fríos" y que "no se centran en lo que (los pacientes) realmente necesitan"32.

Para estudiar este tipo de problemas multifactoriales, se requiere de modelos integradores que permitan examinar la estructura de relaciones entre la diversidad de determinantes de las conductas relevantes al cuidado de la salud. Un modelo que ha resultado ser útil para el estudio de las conductas de salud es el modelo teórico de Betancourt para el "Estudio de la Cultura y
Comportamiento" adaptado para el estudio de la conducta de salud ${ }^{31,33,34}$, el cual postula que las categorías poblacionales, son consideradas como los determinantes más distales de la conducta y son fuentes de variación cultural. Los factores culturales son únicos para la muestra de interés y son más próximos a los factores psicológicos y estos, a su vez, son los determinantes más cercanos a la conducta de salud.

En base a los antecedentes descritos previamente, el objetivo del presente estudio fue examinar el rol y la estructura de relaciones entre los factores socioestructurales, culturales y psicológicos que influyen en las conductas evitativas en salud, tales como falta de motivación para hacer uso de los servicios preventivos, evitación o postergación de citas médicas y rechazo a los profesionales de la salud.

Específicamente, se propone que la discriminación percibida tendría un efecto negativo en las conductas de cuidado de la salud, más aun, se espera que este efecto podría ser directo o a través de las emociones asociadas a la discriminación percibida. Asimismo, se esperaría que la variable cultural "creencias negativas sobre los médicos" tenga una influencia en el cuidado de la salud directamente o indirectamente a través de la discriminación percibida.

Para ello, se investigan las creencias que tienen los cuidadores de niños que asisten a los controles periódicos de salud en la infancia, una actividad que representa el primer acercamiento de la población hacia el sistema de salud $^{32}$, dirigida a promover la salud integral de los niños y detectar anomalías ${ }^{35}$ para derivar oportunamente $^{36}$.

\section{Material y Método}

\section{Participantes}

La muestra estuvo constituida por 337 cuidadores (padres) de niños que asisten a los controles de primera infancia en la Región de La Araucanía. Como criterio de inclusión se consideró que los padres hayan asistido con su hijo al menos a uno de estos controles. El promedio de edad de los participantes fue 31 años (DT $=7,46), 85 \%$ fueron mujeres, $57 \%$ se atendía en el sistema público de salud, $32 \%$ pertenecía a la etnia mapuche, tal como se aprecia en la Tabla 1. 


\section{Instrumentos}

Creencias sobre los médicos: Medida con la subescala de creencias negativas sobre los médi$\cos ^{28}$ que incluye 13 ítems. En ella los participantes deben indicar el grado de acuerdo con los ítems por medio de un formato de respuesta tipo Likert con 5 opciones que van desde $1=$ muy en desacuerdo a $5=$ muy de acuerdo. Esta escala tiene excelente nivel de confiabilidad ( $\alpha=0.96)$.

Discriminación percibida: Medida con un ítem que evalúa la frecuencia de percepción de trato injusto/discriminatorio ${ }^{37}$, con cinco opciones de respuesta desde $1=$ nunca a $5=$ siempre. Donde a mayor puntaje, mayor frecuencia de haber sido tratado injustamente o discriminado.

Emociones y estados afectivos: Se utilizó la Escala de Emociones y Estados Afectivos ${ }^{37}$, la que contiene 13 ítems, en la cual deben informar la intensidad de las emociones y estados afectivos que sintieron como consecuencia del trato injusto. El formato de respuesta es tipo Likert de 5 puntos desde $1=$ nada a $5=$ mucho. Esta escala

Tabla 1. Características de la muestra

\begin{tabular}{|lr|}
\hline Variable & Porcentaje \\
\hline Sexo & \\
$\quad$ Mujer & $85 \%$ \\
Hombre & $15 \%$ \\
\hline Sistema de salud & \\
$\quad$ Público & $57 \%$ \\
$\quad$ Privado & $43 \%$ \\
\hline Etnia & \\
$\quad$ No mapuche & $68 \%$ \\
$\quad$ Mapuche & $32 \%$ \\
\hline Estado civil & \\
$\quad$ Casada/o & $44 \%$ \\
$\quad$ Soltera/o & $30 \%$ \\
Conviviendo & $22 \%$ \\
Nivel educacional & \\
Básica completa & $10 \%$ \\
Media completa o técnica incompleta & $33 \%$ \\
Postgrado & $9 \%$ \\
Ingreso mensual & \\
\$0 - \$500.000 & $64 \%$ \\
\$501.000 - \$1.000.000 & $21 \%$ \\
$\quad \$ 1.000 .001-\$ 1.500 .000$ & $10 \%$ \\
Sobre \$1.500.000 & $5 \%$ \\
\hline
\end{tabular}

Fuente: Elaboración propia. es unifactorial y presenta un muy buen nivel de confiabilidad $(\alpha=0,97)$.

Consecuencias en salud: Evaluadas con la subescala de Consecuencias Evitativas en el Cuidado de la Salud ${ }^{37}$, la cual contiene 7 reactivos, cuyo formato de respuesta es tipo Likert con cinco opciones, que va desde $1=$ muy en desacuerdo a $5=$ muy de acuerdo. Esta subescala presenta un buen nivel de confiabilidad $(\alpha=0,90)$.

Covariables: Se indagó en la etnia (mapuche $=0$, no mapuche $=1$ ), ingreso, nivel educacional, sistema de salud en que se atiende (públi$\mathrm{co}=0$, privado $=1$ ) y la edad de los participantes.

\section{Procedimiento}

Los participantes fueron contactados en los centros de salud familiar (CESFAM) y jardines infantiles previa autorización al Servicio de Salud Municipal de Temuco y de la Junta Nacional de Jardines Infantiles. Las personas interesadas en participar firmaron el consentimiento informado donde se explicitaba el objetivo del estudio y resguardos éticos asociados a esta investigación. Posteriormente, se les aplicó el cuestionario en las salas de espera de los CESFAM y jardines infantiles, lo que demoró alrededor de $30 \mathrm{~min}$. Los participación fueron económicamente retribuidos con $\$ 5$ mil pesos chilenos (aproximadamente USD 7), por el tiempo invertido. Este procedimiento se ajustó a las normas éticas de la Declaración de Helsinki y a los principios éticos de la American Psychological Association. El protocolo fue aprobado por el Servicio de Salud Araucanía Sur.

\section{Análisis estadístico}

Los datos fueron analizados con el software STATA 14.1. Se realizaron análisis de ecuaciones estructurales utilizando el método de estimación de máxima verosimilitud. Este análisis multivariado estudia relaciones complejas entre múltiples variables, algunas de las cuales son medidas $\mathrm{u}$ observadas directamente (ej. indicadores) y otras medidas indirectamente (ej. factores latentes).

En este análisis se evalúa la bondad de ajuste del modelo teórico a los datos, para posteriormente examinar cargas factoriales y coeficientes de regresión. En consecuencia, se utilizaron los siguientes indicadores de bondad de ajuste: chi-cuadrado $\left(\chi^{2}\right)$ no significativo, el Comparative Fit Index (CFI >0,95), el RMSEA (< a 0,08), el índice Tucker-Lewis $($ TLI $>0,95)$ y el SRMR $(<\text { a } 0,05)^{38}$. 


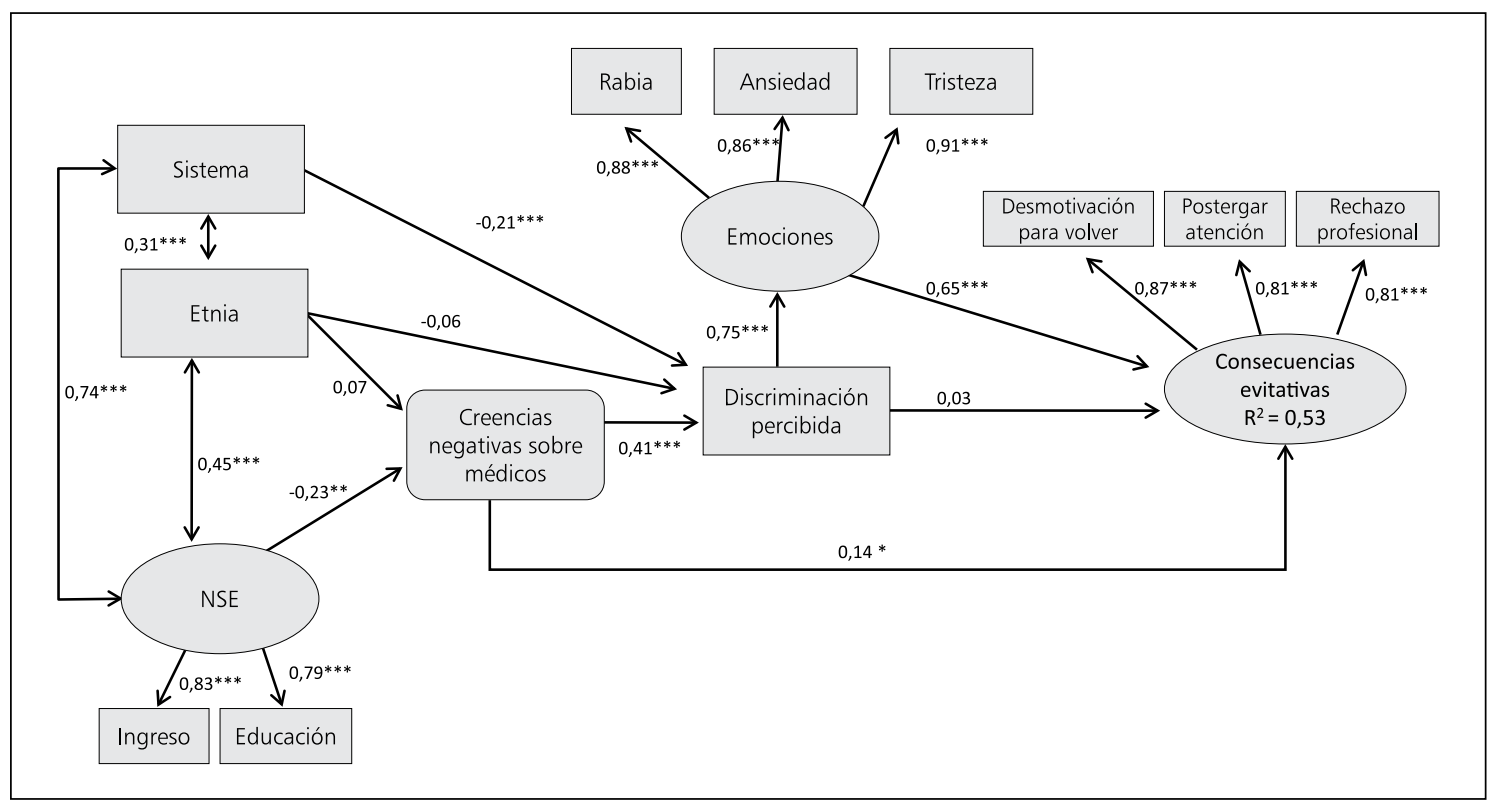

Figura 1. Modelo para creencias sobre los médicos.

\section{Resultados}

El modelo propuesto da cuenta de un muy buen ajuste de los datos $\left(\mathrm{CFI}_{(\mathrm{SB})}=0,98, \chi^{2}[197,46]\right.$ ${ }_{(\mathrm{SB})}=71,32, \mathrm{p}<0,01, \mathrm{RMSEA}_{(\mathrm{SB})}=0,053,[90 \% \mathrm{CI}$ $\left.(0,036-0,082)], \mathrm{TLI}_{(\mathrm{SB})}=0,97, \mathrm{SRMR}_{(\mathrm{SB})}=0,041\right)$, el cual explica el 53\% de la varianza. En la Figura 1 , se observa que solo el nivel socioeconómico tuvo un efecto directo en las creencias negativas sobre los médicos $(\beta=-0,23, \mathrm{p}<0,01)$, es decir, a menor nivel socioeconómico, las creencias fueron más negativas. En relación a la etnia, esta variable no se asoció con las creencias $(\beta=0,07, \mathrm{p}=0,36)$ ni con la discriminación percibida $(\beta=-0,06$, $p=0,29$ ). Respecto del sistema en que se atendía la persona, quienes lo hacían en el sistema público mostraron mayor discriminación percibida $(\beta=-0,21, p<0,001)$ que los que se atendían en el sistema privado.

Por otra parte, las creencias negativas sobre los médicos tuvieron un efecto directo en la discriminación percibida $(\beta=0,41, \mathrm{p}<0,001)$, esto quiere decir que mientras más negativas eran las creencias que tenían las personas sobre los médicos, mayor frecuencia de discriminación percibían. Además, existió una relación directa entre las creencias negativas sobre los médicos y las consecuencias evitativas en el cuidado de la salud, tal que quienes tenían más creencias negativas sobre los médicos, tendieron a tener mayor desmotivación para volver, postergar las atenciones y rechazar a estos profesionales $(\beta=0,14, \mathrm{p}<0,05)$.

Finalmente, se observó que la discriminación percibida no tuvo un efecto directo en las consecuencias antes descritas $(\beta=0,03, p=0,38)$, sino que este efecto estuvo mediado por las emociones negativas asociadas a la discriminación percibida $\left(\beta_{\text {indirecto }}=0,45 ; \mathrm{p}<0,001\right)$, por cuanto, la discriminación percibida se asoció con más emociones negativas $(\beta=0,75, \mathrm{p}<0,001)$ y estas, a su vez, provocaron mayores consecuencias evitativas $(\beta=0,65, \mathrm{p}<0,001)$.

\section{Discusión}

Este estudio tuvo por objetivo demostrar si existe un vínculo entre las creencias negativas sobre los médico y las conductas evitativas del cuidado de la salud, y si esta asociación está mediada por la percepción de discriminación y las emociones negativas derivadas.

En relación al objetivo, se observa una asociación significativa entre creencias y comportamiento evitativos, de modo tal que mientras más 
negativas las creencias sobre los médicos, mayor presencia de conductas tales como el rechazo hacia estos profesionales, postergación de atención médica y desmotivación. Lo anterior confirma lo importante que son las creencias culturales como antecedentes de las conductas evitativas de cuidado de la salud, ya que afectan la obtención de resultados positivos en salud. En consideración que estudios similares se han centrado mayormente en investigar variables tales como la satisfacción y calidad de la atención ${ }^{39}$, este hallazgo contribuye a llenar un vacío en la literatura, respecto al rol que tienen las creencias culturales en los comportamientos de salud.

En relación al rol mediador que la discriminación percibida y las emociones negativas tienen en la asociación entre creencias negativas y conductas evitativas, se observa que la percepción de discriminación no media la relación propuesta, sin embargo, las emociones negativas median el vínculo entre discriminación y consecuencias evitativas. De esta manera, personas que experimentan más rabia, ansiedad y tristeza producto de la discriminación tienden a tener más comportamientos evitativos. Este resultado es consistente con los reportes previos que indican que variables psicológicas, como las emociones y estados afectivos, son los determinantes más directos de la conduc$\mathrm{ta}^{31} \mathrm{y}$ tienen un mayor impacto en la motivación para actuar ${ }^{40}$. De esta forma, y dependiendo del placer/displacer que puedan provocar, tendrían un mayor poder de aproximación o evitación. De esta manera, emociones más negativas tienden a generar más conductas evitativas ${ }^{41}$.

Respecto a las covariables, en este estudio fue posible identificar que mientras mayor es el nivel socioeconómico de las personas, menos negativas son las creencias hacia los profesionales de la salud y que quienes se atienden en el sistema público perciben mayor discriminación. Pese a que la etnia no se asoció con las creencias sobre los médicos, ni la percepción de discriminación, estos hallazgos están en línea con el modelo ${ }^{31,33,34}$ que guía este estudio, y que señala que las variables socioestructurales (covariables) son factores que influyen en la conducta por medio de factores culturales, tales como las creencias socialmente compartidas y variables psicológicas como la percepción de discriminación.

Los resultados de este estudio dan cuenta de la importancia que tiene integrar variables culturales y psicológicas en la comprensión de los comportamientos en salud. Típicamente, se tiende a establecer asociaciones lineales entre variables socioestructurales (nivel socioeconómico, sistema de salud), desconociendo el rol que las variables culturales y psicológicas tienen en los comportamientos en salud. Estudios previos han establecido que existe una relación entre nivel socioeconómico y la educación con desenlaces y conductas de salud, que están mediadas por factores psicológico ${ }^{40} \mathrm{y}$ culturales ${ }^{22}$. Estos hallazgos, además, pueden ser utilizados para el diseño de intervenciones tendientes a disminuir conductas de evitación de cuidado en salud, modificando creencias, y estando atento a las reacciones emocionales que la percepción de discriminación genera en las personas.

Este estudio tiene fortalezas y limitaciones. Con relación a las primeras, cabe destacar el tamaño muestral, número representativo y que permitió los análisis estadísticos realizados. Además, el análisis de modelos de ecuaciones estructurales da cuenta de los efectos directos e indirectos de las variables, y el modelo teórico a la base de este estudio que es utilizado para estudiar la cultura y las conductas en diversos contextos, en este caso de salud.

En relación a las limitaciones, una de ellas fue que la mayoría de los participantes fueron madres, lo cual, si bien es esperable dado el contexto del estudio (control de salud en la primera infancia), no permitió cuantificar las creencias de los hombres, las cuales podrían ser diferentes y, por tanto, determinar conductas de salud distintas. A lo anterior, se suma que en este estudio solo se indagó en creencias negativas sobre los médicos, las cuales pueden coexistir con creencias positivas, incluso podrían moderar el impacto que las creencias negativas tienen en las conductas de salud antes descritas. De esta forma, en futuros estudios, será relevante integrar ambas creencias (positivas y negativas) en un único modelo que facilite la comprensión que la interacción entre ambas puede tener en conductas de salud.

Agradecimientos: Esta investigación fue financiada por CONICYT (Comisión Nacional de Investigación Científica y Tecnológica, Gobierno de Chile), como parte del desarrollo de tesis doctoral de la Dra. María José Baeza-Rivera en la Universidad de La Frontera, número de folio 21140104. 


\section{Referencias}

1. Guanais F, Regalia F, Pérez-Cuevas R, Anaya M, editors. Desde el paciente: Experiencias de la atención primaria de salud en América Latina y el Caribe [Internet]. Inter-American Development Bank; 2018. Available from: https://publications.iadb.org/handle/11319/9041.

2. Ortiz MS, Baeza-Rivera MJ, Salinas-Oñate N, Flynn P, Betancourt $\mathrm{H}$. Atribución de malos tratos en servicios de salud a discriminación y sus consecuencias en pacientes diabéticos mapuche. Rev Med Chile [Internet]. 2016; 144 (10): 1270-6.

3. Desiguales. Orígenes, cambios y desafíos de la brecha social en Chile. Uqbar, Editores. Programa de las Naciones Unidas Para el Desarrollo (PNUD). Santiago de Chile; 2017.

4. Lewis TT, Aiello AE, Leurgans S, Kelly J, Barnes LL. Self-reported experiences of everyday discrimination are associated with elevated C-reactive protein levels in older African-American adults. Brain Behav Immun [Internet]. 2010; 24 (3): 438-43.

5. Szanton SL, Rifkind JM, Mohanty JG, Miller ER, Thorpe RJ, Nagababu E, et al. Racial Discrimination Is Associated with a Measure of Red Blood Cell Oxidative Stress: A Potential Pathway for Racial Health Disparities. Int J Behav Med [Internet]. 2012; 19 (4): 489-95.

6. Brondolo E, Love EE, Pencille M, Schoenthaler A, Ogedegbe G. Racism and Hypertension: A Review of the Empirical Evidence and Implications for Clinical Practice. Am J Hypertens [Internet]. 2011; 24 (5): 51829.

7. Barnes LL, de Leon CFM, Lewis TT, Bienias JL, Wilson RS, Evans DA. Perceived Discrimination and Mortality in a Population-Based Study of Older Adults. Am J Public Health [Internet]. 2008; 98 (7): 1241-7.

8. McLaughlin KA, Hatzenbuehler ML, Keyes KM. Responses to Discrimination and Psychiatric Disorders Among Black, Hispanic, Female, and Lesbian, Gay, and Bisexual Individuals. Am J Public Health [Internet]. 2010; 100 (8): 1477-84.

9. Soto JA, Dawson-Andoh NA, BeLue R. The relationship between perceived discrimination and Generalized Anxiety Disorder among African Americans, Afro Caribbeans, and non-Hispanic Whites. J Anxiety Disord [Internet]. 2011; 25 (2): 258-65.

10. Levine DS, Himle JA, Abelson JM, Matusko N, Dhawan N, Taylor RJ. Discrimination and Social Anxiety Disorder Among African-Americans, Caribbean Blacks, and Non-Hispanic Whites. J Nerv Ment Dis [Internet]. 2014; 202 (3): 224-30.

11. Marinho ML. Health Inequity in a Neoliberal Society:
Lifestyle Choices or Constrained Practices? Warwick University; 2015.

12. Bird ST, Bogart LM, Delahanty DL. Health-Related Correlates of Perceived Discrimination in HIV Care. AIDS Patient Care STDS [Internet]. 2004; 18 (1): 19-26.

13. Smedley BD, Myers HF. Conceptual and Methodological Challenges for Health Disparities Research and Their Policy Implications. J Soc Issues 2014; 70 (2): 382-91.

14. Burgess D, Ding Y, Hargreaves M, van Ryn M, Phelan S. The Association between Perceived Discrimination and Underutilization of Needed Medical and Mental Health Care in a Multi-Ethnic Community Sample. J Health Care Poor Underserved [Internet]. 2008; 19 (3): 894-911.

15. Hausmann LRM, Jeong K, Bost JE, Ibrahim SA. Perceived Discrimination in Health Care and Health Status in a Racially Diverse Sample. Med Care [Internet]. 2008; 46 (9): 905-14.

16. Trivedi AN, Ayanian JZ. Perceived discrimination and use of preventive health services. J Gen Intern Med [Internet]. 2006; 21 (6): 553-8.

17. Crawley LM, Ahn DK, Winkleby MA. Perceived Medical Discrimination and Cancer Screening Behaviors of Racial and Ethnic Minority Adults. Cancer Epidemiol Biomarkers Prev [Internet]. 2008; 17 (8): 1937-44.

18. Moody-Ayers SY, Stewart AL, Covinsky KE, Inouye SK. Prevalence and Correlates of Perceived Societal Racism in Older African-American Adults with Type 2 Diabetes Mellitus. J Am Geriatr Soc [Internet]. 2005; 53 (12): 2202-8.

19. Casagrande SS, Gary TL, LaVeist TA, Gaskin DJ, Cooper LA. Perceived Discrimination and Adherence to Medical Care in a Racially Integrated Community. J Gen Intern Med [Internet]. 2007; 22 (3): 389-95.

20. Brener L, Von Hippel W, Von Hippel C, Resnick I, Treloar C. Perceptions of discriminatory treatment by staff as predictors of drug treatment completion: Utility of a mixed methods approach. Drug Alcohol Rev [Internet]. 2010; 29 (5): 491-7.

21. Bazargan-Hejazi S, Bing E, Bazargan M, Der-Martirosian C, Hardin E, Bernstein J, et al. Evaluation of a Brief Intervention in an Inner-City Emergency Department. Ann Emerg Med [Internet]. 2005; 46 (1): 67-76.

22. Amador JA, Flynn PM, Betancourt H. Cultural beliefs about health professionals and perceived empathy influence continuity of cancer screening following a negative encounter. J Behav Med 2015; 38 (5): 798 808.

23. De Leersnyder J, Boiger M, Mesquita B. Cultural regulation of emotion: individual, relational, and structural sources. Front Psychol [Internet]. 2013; 4. 
24. Flynn PM, Betancourt H, Ormseth SR. Culture, Emotion, and Cancer Screening: an Integrative Framework for Investigating Health Behavior. Ann Behav Med [Internet]. 2011; 42 (1): 79-90.

25. Betancourt H, Flynn P, Ormseth S. Healthcare Mistreatment and Continuity of Cancer Screening Among Latino and Anglo American Women in Southern California. Women Health 2011; 51 (1): 1-24.

26. Flynn PM, Betancourt H, Garberoglio C, Regts GJ, Kinworthy KM, Northington DJ. Attributions and emotions regarding health care mistreatment impact continuity of care among Latino and Anglo American women. Cult Divers Ethn Minor Psychol [Internet]. 2015; 21 (4): 593-603.

27. Baeza-Rivera $M$, Betancourt $H$, Salinas-Oñate $N$, Ortiz MS, Marín O, Valle G. Identificación y medición de creencias culturales sobre los médicos: construcción y validación de un instrumento. Rev Med Chile 2018; 146: 308-14.

28. Moy B, Park ER, Feibelmann S, Chiang S, Weissman JS. Barriers to repeat mammography: cultural perspectives of African American, Asian, and Hispanic women. Psycho Oncology 2006; 15 (7): 623-34.

29. Buki LP, Borrayo EA, Feigal BM, Carrillo IY. Are All Latinas the Same? Perceived Breast Cancer Screening Barriers and Facilitative Conditions. Psychol Women Q [Internet]. 2004; 28 (4): 400-11.

30. Betancourt H, Flynn P, Riggs M, Garberoglio C. A cultural research approach to instrument development: the case of breast and cervical cáncer screening among Latino and Anglo women. Heal Educ Res 2010; 25 (6): 991-1007.

31. Alarcón AM, Vidal AC. Dimensiones culturales en el proceso de atención primaria infantil: perspectivas de las madres. Salud Publica Mex 2005; 47 (6): 440-6.

32. Betancourt H, Flynn PM. The psychology of health:
Physical health and the role of culture in behavior. In: Handbook of US Latino psychology: Developmental and community-based perspectives. 2009. p. 347-61.

33. Betancourt H, Flynn PM, Ormseth SR. Healthcare Mistreatment and Continuity of Cancer Screening Among Latino and Anglo American Women in Southern California. Women Health [Internet]. 2011; 51 (1): 1-24.

34. MINSAL. Norma técnica para la supervisión de niños y niñas de 0 a 9 años en la atención primaria de salud. 2013.

35. Szot J. Reseña de la salud pública materno-infantil chilena durante los últimos 40 años: 1960-2000. Rev Chil Obstet Ginecol 2002; 67 (2): 129-35.

36. Baeza-Rivera M. Discriminación percibida y sus consecuencias en las conductas de cuidado de la salud: Un estudio multifactorial. Universidad de La Frontera; Temuco, 2015.

37. $\mathrm{Hu} \mathrm{L}$, Bentler PM. Cutoff criteria for fit indexes in covariance structure analysis: Conventional criteria versus new alternatives. Struct Equ Model A Multidiscip J [Internet]. 1999; 6 (1): 1-55.

38. Pezoa M. Satisfacción y Calidad Percibida en la Atención de Salud Hospitalaria. Superintendencia de Salud. Santiago de Chile; 2013.

39. Savani K, Alvarez A, Mesquita B, Markus HR. Feeling close and doing well: The prevalence and motivational effects of interpersonally engaging emotions in Mexican and European American cultural contexts. Int J Psychol [Internet]. 2013; 48 (4): 682-94.

40. Russell JA. A circumplex model of affect. J Pers Soc Psychol [Internet]. 1980; 39 (6): 1161-78.

41. Kan C, Kawakami N, Karasawa M, Love GD, Coe CL, Miyamoto Y, et al. Psychological Resources as Mediators of the Association Between Social Class and Health: Comparative Findings from Japan and the USA. Int J Behav Med [Internet]. 2014; 21 (1): 53-65. 